\title{
Very High Bit Rate Power Line Communications For Home Networks
}

\author{
Ali Maiga, Jean-Yves Baudais and Jean-François Hélard \\ Institute of Electronics and Telecommunications of Rennes \\ 35043 Rennes Cedex, France \\ Email: \{ali.maiga, jean-yves.baudais, jean-francois.helard\}@insa-rennes.fr
}

\begin{abstract}
Power line communications (PLC) has become a viable local area network (LAN) solution for in-home networks. PLC systems can be used in a wide variety of applications requiring high data rate. Therefore, there is a need to achieve an extremely high bit rate. In this paper, the increase in current technology bandwidth up to $100 \mathrm{MHz}$ is investigated. Furthermore, a fast computational resource allocation algorithm is also developed for linear precoded discrete multitone (LP-DMT), which enables reliable high bit rate under peak BER constraint. Simulations are run over PLC channels and it is highlighted that $1 \mathrm{Gbit} / \mathrm{s}$ transmission is achievable and it is also shown that peak BER constraint algorithms give better performance compared to classical peak SER constraint algorithms. In addition, the linear precoding technique brings significant bit rate gain.
\end{abstract}

\section{INTRODUCTION}

Initially developed in the 1980's for low bit rate applications, such as telemetry, infrastructure control and consumption measurement, powerline communication (PLC) technologies have recently drawn an increasing interest within the scientific community for high bit rate communications over the power grid. Due to significant advances achieved in terms of modulation and signal processing schemes on one hand, and owing to the ubiquity of the outlet on the other hand, PLC is today considered as a solution of high potential for indoor applications and constitutes a convenient and cheap alternative to already existing technologies.

We are witnessing an increase in data applications with high bandwidth requirements and the number of in-home devices in PLC systems (Fig. 1). Therefore, the demand for multimedia services such as video and audio conferencing, online training, news, software distribution and database replication is increasing. Achieving an extremely high bit rate is needed in order to guarantee the different qualities of service (QoS). The FP7 OMEGA (Home Gigabit Networks) project targets this goal and is devoted to develop innovations in transmission technology and convergence layer for wireless i.e., LAN, ultra wide band (UWB), $60 \mathrm{GHz}$ systems, visible light communication systems and PLC systems [1], [2].

The powerline channel exhibits multipaths caused by reflections on the discontinuities of the network and offers impulse responses that can be assumed as quasi-static. These two main features encourage the use of robust communication systems for the former and the exploitation of the knowledge of the channel state information (CSI) at the transmitter side for the latter. Under this assumption, adaptive bit loading princi-

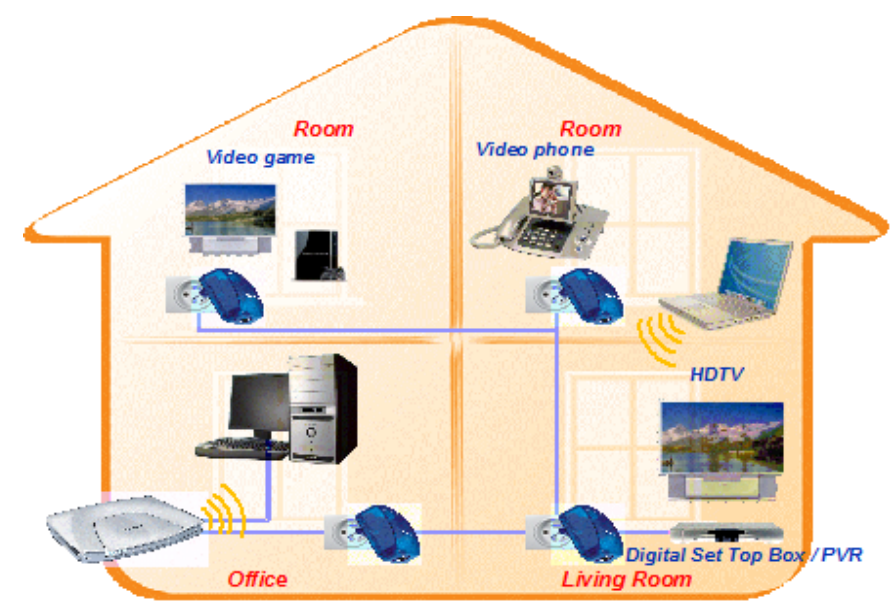

Fig. 1. Illustration of some indoor applications - OMEGA [3].

ples can advantageously be applied using discrete multitone modulation (DMT) in the PLC context [4]. Many loading algorithms for allocating power and bits have been developed and are based on the well known waterfilling approach. The first proposed [5] algorithm for xDSL applications reaches the optimal solution using a greedy algorithm but leads to intensive computational complexity when the number of bits to be transmitted per DMT symbol is large. Many suboptimal algorithms with less computational complexity have since been developed (refer, for example, [6] and references inside for an extensive overview).

In [6], bit-loading algorithm with symbol error rate (SER) has been proposed for Homeplug AV (HPAV) systems which operate in the frequencies lower than $30 \mathrm{MHz}$. Owing to electromagnetic compatibility constraint, a power spectral density (PSD) mask is defined and only 917 usable subcarriers (tones) are used in order not to interfere with amateur radios [7], [8]. In addition,modulation densities from BPSK to 1024 QAM are independently applied to each subcarrier based on the link budget between the transmitter and the receiver. Consequently, all these limitations reduce significantly the total bit rate. So far, HPAV solution offers a theoretical $220 \mathrm{Mbps}$ bit rate over the physical (PHY) layer (assuming 1024 QAM modulation on all subcarriers).

In [9], [10], the proposed bit-loading algorithms try to maximize the overall PLC throughput under a peak bit er- 
ror rate (BER) constraint. As in power constraint, a peak constraint is defined in opposition to average constraint [11]. The peak power constraint is the PSD constraint where the power is limited for each subcarrier. In the case of BER or SER, this constraint is applied to each bit or symbol. In the proposed algorithms, the idea is to iteratively allocate bits until satisfaction of the peak BER constraint. Although these algorithms give good results, their computational complexity is still rather high at low SNR values. What is needed is an algorithm that accurately determines the final bit allocation in a low computational complexity fashion. In [12], it has been shown that when increasing the bandwidth up to $100 \mathrm{MHz}$, the capacities of various in-home measured channels reach at least $1.12 \mathrm{Gbit} / \mathrm{s}$.

In this paper, the enhancement of current technology bandwidth up to $100 \mathrm{MHz}$ is investigated where the maximum number of bits per symbol is set to 15 bits as in xDSL systems. To achieve high bit rate, a fast computational resource allocation which tries to maximize the overall bit rate under peak BER constraint is proposed. This new algorithm dynamically allocates subcarriers and bits based on the linear precoding technique. It is much simpler to implement, since no convergence iterations are required, but simply a look-up table is used, in order to store, for each allowable modulation order, the required channel conditions to guarantee a target BER. Due to power spectral density (PSD) constraint in PLC systems, there is the same peak power constraint on each subcarrier and there is no power allocation. Linear precoded DMT (LPDMT) is a combination of multi-carrier and spread spectrum techniques also known as MC-SS techniques. This technique has shown very good performances in difficult environments and brings a significant increase in bit rate compared to classical DMT systems [13], [14].

This paper is organized as follows. Section II presents the rate adaptive algorithms which have been applied in the literature for resource allocation under peak SER constraint with classical DMT systems and LP-DMT systems. Section III gives the new proposed resource allocation algorithms under peak BER constraint. Section IV describes the studied PLC systems. The performance of the proposed algorithms over PLC channels is given in section V. Finally, section VI concludes the paper.

\section{Peak SER constraint Resource allocation SCHEMES}

\section{A. Classical DMT systems}

The term DMT denotes orthogonal frequency division multiplex (OFDM) based communication systems that adapt the transmission to the channel conditions individually for each subcarrier, by means of so called bit-loading. This technique is currently used for xDSL solutions and HPAV. The number of bits on each subcarrier depends on the signal-to-noise ratio (SNR) of the subcarrier and the peak SER constraint approximated by the normalized SNR (also known as SNR gap) $\Gamma$. Hence, in peak power constraint, the total achievable bit rate per OFDM symbol is

$$
R_{\mathrm{DMT}}=\sum_{n=1}^{N} R_{n}=\sum_{n=1}^{N}\left\lfloor\log _{2}\left(1+\frac{1}{\Gamma}\left|H_{n}\right|^{2} \frac{E}{N_{0}}\right)\right\rfloor,
$$

where $N$ is the number of subcarriers, $\left|H_{n}\right|^{2}$ is the frequency channel coefficient of subcarrier $n$. All subcarriers have the same peak power constraint $E$ and there is no power allocation.The energy used by each subcarrier is given by

$$
E_{n}=\left(2^{R_{n}}-1\right) \frac{\Gamma N_{0}}{\left|H_{n}\right|^{2}}=E-\epsilon_{n} \leq E .
$$

This energy is lower than the PSD constraint, therefore there is a loss of residual $\epsilon_{n}$ energy per subcarrier. The total loss of energy is the sum $\sum_{n=1}^{N} \epsilon_{n}$. For mutual exploitation of residual energies $\epsilon_{n}$, it has been proposed to add a linear precoding component to classical DMT [10], [13], [14].

\section{B. Linear precoded DMT systems}

As previously stated, LP-DMT results from the combination of multi-carrier modulation and spread spectrum. In our study, the LP component is not used to share access between users, as code division multiplex access (CDMA) does, but instead to multiplex different data symbols of a given user. The merging process consists in connecting a set of subcarriers with precoding sequences to mutually exploit their energies. This set of subcarriers is called block in the following and the subcarriers are not necessary adjacent. The number of blocks is defined as the ratio of total number of subcarriers $N$ to the precoding sequence length $L$. If judiciously done, each resulting set holds an equivalent SNR such that the total supported throughput is greater than the sum of the individual throughputs supported by each subcarrier taken separately. Fig. 2 gives the LP-DMT transmitter-receiver model. From the classical DMT systems, the linear precoding matrix which is a Hadamard matrix is added.

In a general approach, for LP-DMT systems, the data have to be distributed into the frames in the time or frequency domain or both [13]. This data distribution is commonly called chip-mapping and can be designed in an adaptive manner according to a given resource allocation strategy. Therefore, in LP-DMT, bits, subcarriers, energies as well as precoding sequences have to be allocated in order to optimize the selected goal.

Over the block $S$ of $L$ subcarriers, the optimum achieved bit rate per OFDM symbol under PSD constraint, with ZF (zero forcing) receiver and unconstrained modulations is [13]

$$
R_{S}=L \times \log _{2}\left(1+\frac{E}{\Gamma N_{0}} \frac{L}{\sum_{n \in S} \frac{1}{\left|H_{n}\right|^{2}}}\right) .
$$

For real systems, the bit rate achieved by an adaptive LP-DMT system using discrete modulation is maximized if, on block $S, b_{i}$ bits are allocated to precoding sequence $c_{i}$, and $b_{i}$ is 


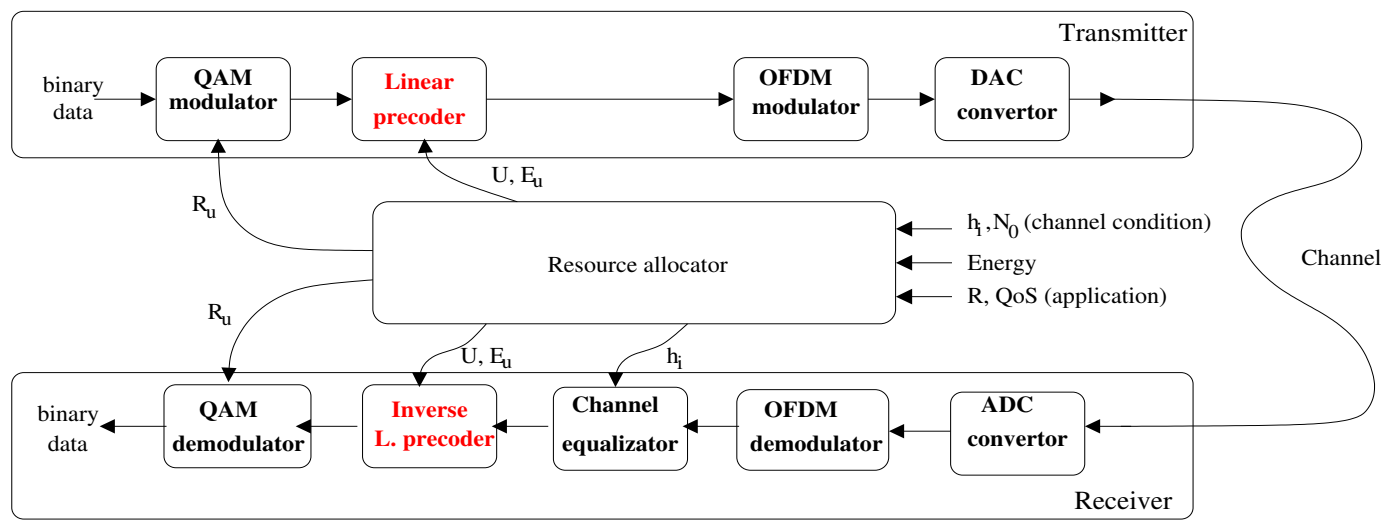

Fig. 2. Transmitter-receiver model.

expressed as [10]

$$
b_{i}= \begin{cases}\left\lfloor R_{S} / L\right\rfloor+1 & \left(1 \leq i \leq n_{c}\right), \\ \left\lfloor R_{S} / L\right\rfloor & \left(n_{c}<i \leq L\right),\end{cases}
$$

where

$$
n_{c}=\left\lfloor L\left(2^{R_{S} / L-\left\lfloor R_{S} / L\right\rfloor}-1\right)\right\rfloor .
$$

Then, the achievable bit rate per OFDM symbol $\overline{R_{S}}$ is

$$
\overline{R_{S}}=n_{c} \times\left(\left\lfloor R_{S} / L\right\rfloor+1\right)+\left(L-n_{c}\right) \times\left\lfloor R_{S} / L\right\rfloor .
$$

Each precoding sequence energy contribution in the total energy of the given block, is given by

$$
E_{i}=\left(2^{b_{i}}-1\right) \frac{\Gamma N_{0}}{L^{2}} \sum_{n \in S} \frac{1}{\left|H_{n}\right|^{2}},
$$

which satisfies

$$
\sum_{i} E_{i} \leq E
$$

\section{A NEW PEAK BER CONSTRAINT RESOURCE ALLOCATION SCHEME}

In this paper, the overall throughput under peak BER constraint per subcarrier is maximized. In (1) and (3), the SNR gap $\Gamma$ is defined for a given target SER with uncoded QAM and has a constant value for all the modulation orders. In a coded QAM case, the coding gain is taken into account with a modified expression of $\Gamma$ [4]. For a constant peak BER constraint per subcarrier, $\Gamma$ is no more constant for all the modulation orders as the SER constraint is approximated by

$$
\mathrm{SER} \approx b_{i} \times \mathrm{BER},
$$

where $b_{i}$ is the modulation order. Therefore, bit-loading algorithms have to be performed with variable SNR gap $\Gamma_{b_{i}}$ for each modulation order and $\Gamma_{b_{i}}$ is approximated by

$$
\Gamma_{b_{i}} \approx \frac{1}{3}\left(Q^{-1}\left(\frac{b_{i} \times \mathrm{BER}}{4}\right)\right)^{2},
$$

where $Q^{-1}$ is the inverse of the well-known $\mathrm{Q}$-function given by:

$$
Q(\alpha)=\frac{1}{\sqrt{2 \pi}} \int_{\alpha}^{+\infty} e^{-\frac{x^{2}}{2}} d x
$$

In [9], [10] and [16], it has been proposed bit-loading algorithms trying to maximize the overall throughput under a peak BER constraint per subcarrier. These algorithms iteratively try to converge to an almost optimum allocation; the number of iterations, that needs to be minimized in order to limit the computational complexity, is obviously a crucial point of the algorithms. The following proposed algorithm determines the final bit allocation without iterations on the number of bits.

\section{A. Proposed bit-loading algorithm with peak BER constraint}

Note that the DMT system can be obtained from the LPDMT system with a precoding sequence length $L=1$. A useful algorithm based on linear precoding technique is developed in a single user context. The main idea is to predefine the sum of inverse channel gains within a block $S$ which is needed to transmit $R_{S}$ bits. From (3), the needed sum of inverse channel gains (NSICG) is derive as

$$
\mathrm{NSICG}_{b}=\frac{E}{\Gamma_{b} N_{0}} \frac{L}{\left(2^{b}-1\right)},
$$

when assuming the same modulation order $b$ for all precoding sequences within the block $S$, and $\Gamma_{b}$ is computed using (10).

Theorem 1: For a given constant peak BER, the function NSICG is a decreasing function of $b$.

Proof: The proof is obvious since $Q($.$) is a monoton-$ ically decreasing function. Therefore, its inverse $Q^{-1}($.$) is$ an increasing function. Since the function $b \mapsto\left(2^{b}-1\right)$ is an increasing function of $\mathrm{b}$, it is derived that $\mathrm{NSICG}_{b}$ is a decreasing function of $b$.

Let $b=\left\lfloor R_{S} / L\right\rfloor$ for the block $S$ of $L$ subcarriers. Therefore, $b \leq R_{S} / L<b+1$ and then,

$$
\frac{E}{\Gamma_{b} N_{0}} \frac{L}{\left(2^{b}-1\right)} \leq \frac{E}{\Gamma N_{0}} \frac{L}{\left(2^{\frac{R_{S}}{L}}-1\right)}<\frac{E}{\Gamma_{b+1} N_{0}} \frac{L}{\left(2^{b+1}-1\right)} \text {. }
$$

From (3), it is derived that

$$
\mathrm{NSICG}_{b} \leq \sum_{n \in S} \frac{1}{\left|H_{n}\right|^{2}}<\mathrm{NSICG}_{b+1} .
$$

Hence, for the block $S$, the achieved bit rate is computed from (3) setting $\Gamma=\Gamma_{b}$. The bits allocated to precoding 
sequences cannot be computed using (4) because $n_{c}$ in (5) was calculated for a constant SNR gap $\Gamma$ for all modulation orders. When rewriting the PSD constraint (8), the new $n_{c}$ is derived as

$$
n_{c}=\left\lfloor\frac{L\left(2^{R_{S} / L}-2^{\left\lfloor R_{S} / L\right\rfloor}\right)}{\left(2^{\left\lfloor R_{S} / L\right\rfloor+1}-1\right) \frac{\Gamma_{b+1}}{\Gamma_{b}}-\left(2^{\left\lfloor R_{S} / L\right\rfloor}-1\right)}\right\rfloor,
$$

and it can be easily shown that $n_{c} \leq L$. Hence, the bit allocated to each precoding sequence can be computed using (4). The bit-loading algorithm for a block $S$ of $L$ subcarriers is given below:

1: for each modulation order $b, b=b_{\min }, \ldots, b_{\max }$ do

2: $\quad$ compute $\Gamma_{b}$ from (10)

3: $\quad$ compute $\mathrm{NSICG}_{b}$ from (12)

4: end for

5: for the block $S$ do

6: $\quad$ compute $s=\sum_{n \in S} 1 /\left|H_{n}\right|^{2}$

7: $\quad$ if $s<\mathrm{NSICG}_{b_{\max }}$ then

8: $\quad b_{i}=b_{\max }$ for all $i$

9: $\quad$ else

10: $\quad$ find $b$ as $\mathrm{NSICG}_{b} \leq s<\mathrm{NSICG}_{b+1}$

11: $\quad$ compute $R_{S}$ using (3) and setting $\Gamma=\Gamma_{b}$

12: $\quad$ compute $n_{c}, b_{i}$ and $E_{i}$

13: end if

14: end for

This algorithm is much simpler to implement, since no convergence iterations are required, but simply a look-up table in order to store, for each allowable modulation order $b$, the required $\Gamma_{b}$ and $\mathrm{NSICG}_{b}$ to guarantee a target BER. For multi block systems, the aforementioned bit-loading algorithm is applied for each block. Subcarriers that maximize the overall throughput are then chosen. It has been proven that choosing the best available subcarriers for each block maximizes the system throughput [13].

\section{SYSTEMS DESCRIPTION}

Homeplug AV [7] has been standardized by Homeplug Powerline Alliance in order to improve the previous Homeplug 1.0 standard. While Homeplug 1.0 was designed to distribute the Internet access, HPAV aims at supporting Audio/Video as well as data traffic within home. HPAV employs the advanced PHY and MAC technologies and provides up to the PHY rate of $220 \mathrm{Mbps}$. It uses the same MAC protocol of CSMA/CA as Homeplug 1.0. The OFDM modulation is performed with a 3072-FFT size, resulting in carrier spacing of $24.414 \mathrm{kHz}$ and only 917 carriers are used for modulation in North America. In addition, to achieve signaling bit rates near theoretical capacity, HPAV specifications prescribe the use of turbo convolutional codes for forward error-correction coding.

The extended PLC system studied within the European ICT FP7 OMEGA project will focus on developing a wide band (up to $100 \mathrm{MHz}$ ) transmission interface that allows for coexistence and compatibility with the existing HPAV specification. Therefore, the same carrier spacing is kept as HPAV for this

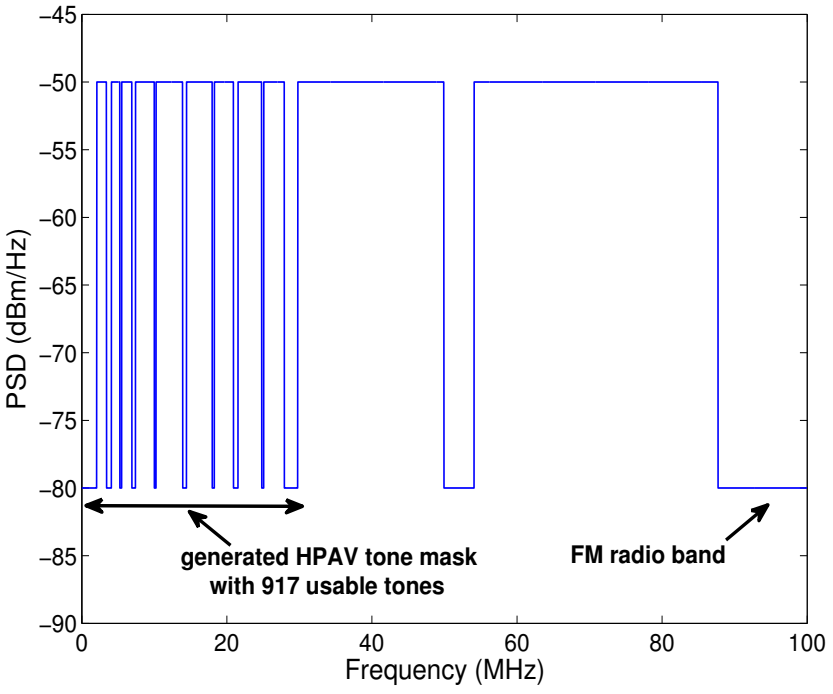

Fig. 3. Extended PLC PSD mask for US.

new system. The OFDM modulation is performed with a $8192-$ FFT size, resulting in the same carrier spacing as in HPAV. Compared to HPAV, the modulation densities are increased up to 32768 QAM (15 bits). Taking into account the amateur radio and FM radio bands up to $100 \mathrm{MHz}$, a PSD tone mask (Fig. 3) as in HPAV is performed, where 4 or 5 additional subcarriers on either side of the each notch are set to zero amplitude in order to guarantee that the energy inside the licensed band will be at least $30 \mathrm{~dB}$ lower than the normal transmit power [15]. Then, there are 3116 usable subcarriers including the 917 subcarriers of HPAV.

Detecting or not HPAV, four scenarios given in table I can be considered with respect to proposed PSD mask.

\begin{tabular}{c|cccc}
\hline System & HPAV & Extended HPAV & Full DMT & Full LP-DMT \\
\hline Bandwidth & $30 \mathrm{MHz}$ & $100 \mathrm{MHz}$ & $100 \mathrm{MHz}$ & $100 \mathrm{MHz}$ \\
Modulation & DMT & DMT & DMT & LP-DMT \\
Constraint & SER & SER & BER & BER \\
max. QAM & $2^{10}$ & $2^{15}$ & $2^{15}$ & $2^{15}$ \\
\hline
\end{tabular}

TABLE I

POSSIBLE UTILIZATIONS OF THE BANDWIDTH.

\section{Simulation Results}

In this section, simulation results for proposed resource allocation schemes are presented for the new PLC systems with the characteristics summarized in table I. Perfect synchronization and channel estimation are assumed. A high background noise level of $-110 \mathrm{dBm} / \mathrm{Hz}$ is assumed and the signal is transmitted with respect to a flat PSD of $-50 \mathrm{dBm} / \mathrm{Hz}$ with notches specified in Fig. 3. Results are given for a fixed target BER of $10^{-5}$. A constant coding gain $\gamma_{c}$ of $6 \mathrm{~dB}$ is assumed for all modulation orders and the noise margin $\gamma_{m}$ is set to $3 \mathrm{~dB}$. In order to take into account the coding and 


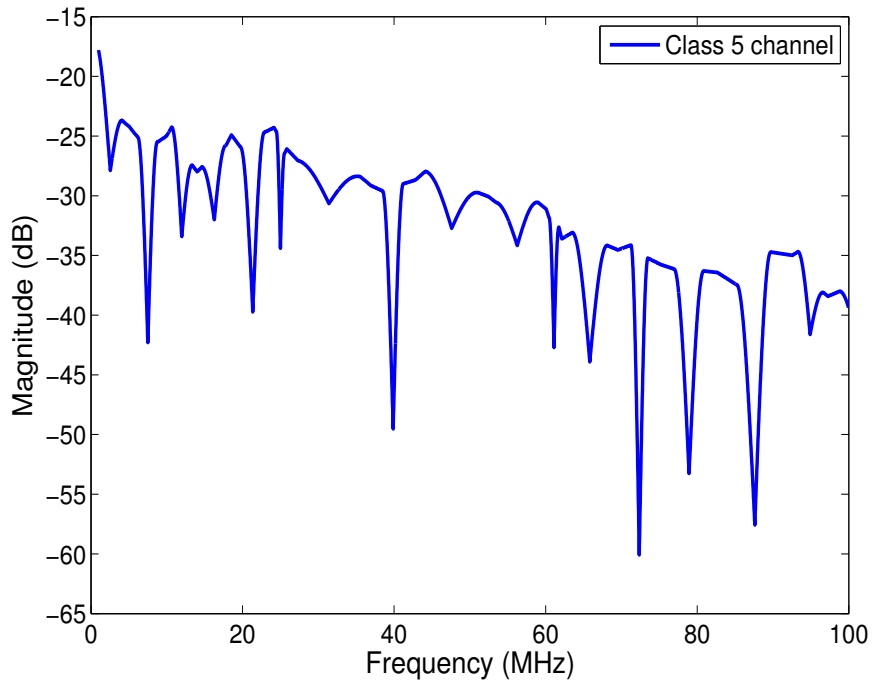

Fig. 4. Illustration of class 5 channel transfert function.

margin gain, the global SNR gap $\Gamma_{b}^{\mathrm{g}}$ is given by [4]:

$$
\Gamma_{b}^{\mathrm{g}}=\Gamma_{b}-\gamma_{c}+\gamma_{m}(d B),
$$

where $\Gamma_{b}$ is computed from (10). The maximum number of bits per symbol is limited to 15 . The multipath channel model given in [12] for indoor PLC is used. In [12], PLC channels are classified into 9 classes according to their capacities, and a model of transfer function is associated to each class. The higher the channel class number, the better its channel conditions. Fig. 4 shows the transfer function of class 5 channel.

In a first step, the impact of the enhancement of the bandwidth up to $100 \mathrm{MHz}$ with peak BER constraint bit loading is analyzed. First simulations are run over the class 5 channel and the precoding sequence length is equal to 4 or 32 . Fig. 5 depicts the achieved bit rates for different bit loading algorithms when the channel gain varies. The difference between the HPAV and the "Extended HPAV" curves confirms the fact that the extended bandwidth system significantly increases the total bit rate. This result is highlighted in Fig. 8 for different PLC channel classes. According to "Extended HPAV" and "Full DMT" curves, it is observed that bit loading with peak BER constraint (variable SNR gap) gives better results than bit loading with peak SER constraint (constant SNR gap). In addition, the linear precoding component ("Full LP-DMT") brings a significant additional bit rate gain and this gain is more important for a longer precoding sequence. Fig. 6 gives the additional gain of proposed bit-loading algorithms with peak BER constraint compared to the "Extended HPAV" system with peak SER constraint. For an average channel gain of $-48 \mathrm{~dB}$, the "Full LP-DMT" system with a precoding sequence length equal to 32 offers a bit rate percentage increase of about $11 \%$ compared to the "Extended HPAV" system. The reason for the better performance of the LPDMT system is explained in Fig. 7, where the used energy of different algorithms are compared. Fig. 7 gives the used energy

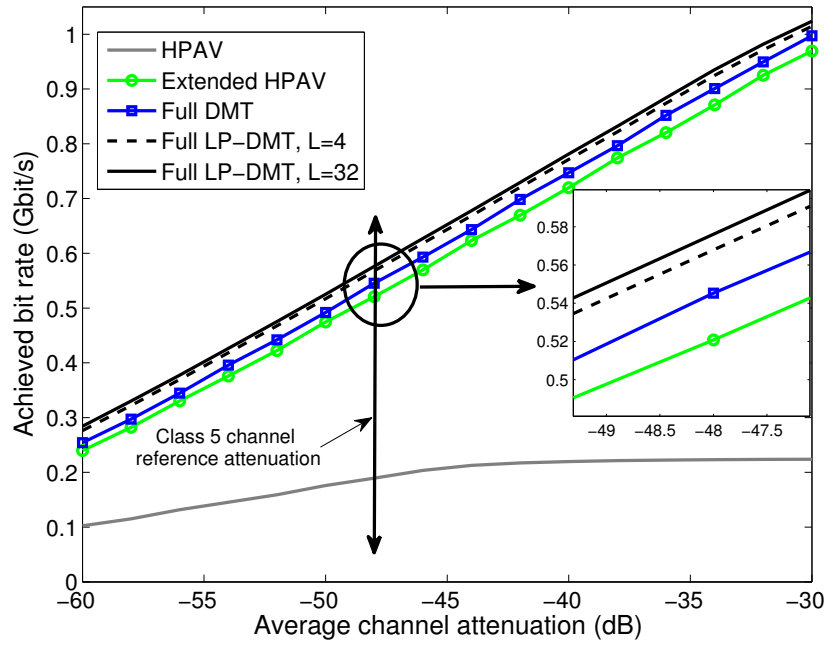

Fig. 5. Achieved bit rates for constant and variable SNR gap for class 5 channel.

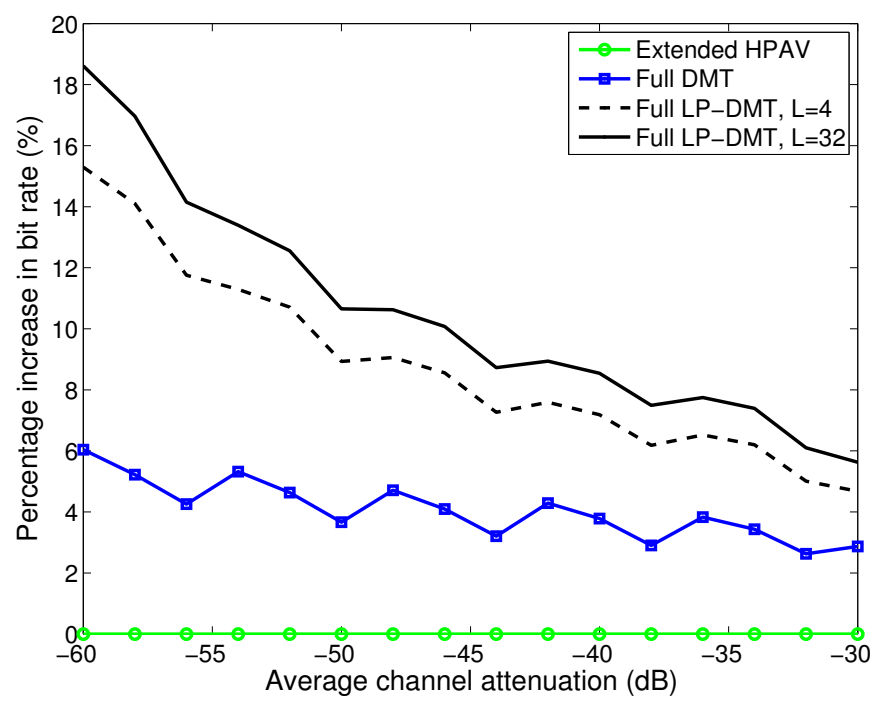

Fig. 6. Percentage increase in bit rate compared to Extended HPAV.

per subcarrier for only 2500 subcarriers. To highlight the energy distribution, these subcarriers are sorted in descending order. The used energy is the minimal required energy allowing the transmission of the maximum data rate. For example, with DMT system, this used energy is given by $E_{n}$ (2). Jump positions in curves correspond to the change in modulation orders. It is clear that the DMT algorithms in "Extended HPAV" and "Full DMT" systems are not fully exploiting the available energy on each subcarrier due to discrete modulation orders. The used energy of both systems are similar and the gap between the curves are due to different constraints (peak SER and peak BER). The precoding component accumulates the residual energies $\epsilon_{n}$ (2) of a given block of subcarriers to transmit additional bits. The adaptive LP-DMT system utilizes more efficiently the PSD limit. The longer the precoding 


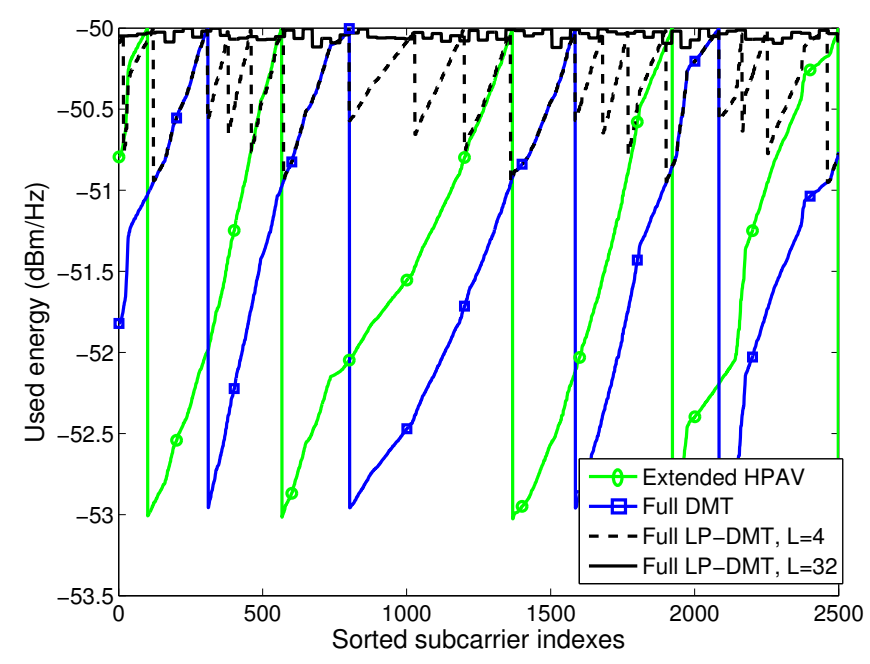

Fig. 7. Used energy for class 5 channel at its reference gain value of $-48 \mathrm{~dB}$.

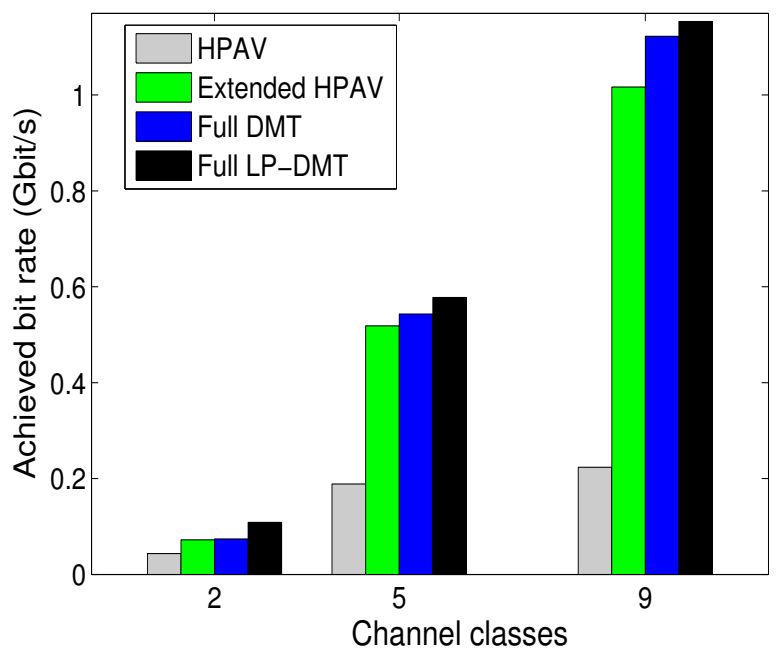

Fig. 8. Achieved bit rate for class 2, 5 and 9 channels.

sequence, the better the used energy. However, a compromise on precoding sequence length has to be made because the self interference (interference between precoding sequences) increases with the precoding sequence length, due to channel selectivity.

In the second step, results are given for different channel classes and only three channel classes (class 2, 5 and 9) are considered. The bit-loading algorithms are performed for the systems stated in section IV. Fig. 8 shows the achieved bit rate for class 2, 5 and 9 channels in single user context. As previously stated, when extending the bandwidth (up to $100 \mathrm{MHz}$ ), the bit rate is significantly improved compared to HPAV system. The total bit rate reaches the gigabit per second in the best channel conditions. This figure confirms the fact that the linear precoding based system (Full LP-DMT) gives best performance whatever the considered channel class.

\section{CONCLUSION}

In this paper, the achievement of very high bit rate has been investigated for powerline communications for home networks. Increased current technology bandwidth up to $100 \mathrm{MHz}$ combined with new modulation schemes allow obtaining a bit rate of $1 \mathrm{Gbit} / \mathrm{s}$. A fast computational resource allocation under fix peak BER constraint has been proposed. It has been shown through simulations that the proposed method combined with linear precoding component gives best performances in PLC context.

\section{ACKNOWLEDGMENT}

The research leading to these results has received funding from the European Community's Seventh Framework Programme FP7/2007-2013 under grant agreement $n^{\circ} 213311$ also referred to as OMEGA.

\section{REFERENCES}

[1] ICT OMEGA project, http://www.ict-omega.eu

[2] J.P. Javaudin, M. Bellec, "Gigabit Home Networks," OMEGA ICT Project, proc. of ICT 2008.

[3] OMEGA project deliverable D3.1 "State of the art, application scenario and specific requirements for PLC," www.ict-omega.eu/fileadmin/documents/deliverables/OmegaD3.1.pdf.

[4] J. M. Cioffi,“A multicarrier primer,” November 1991, ANSI Contribution T1E1.4/91-157, Clearfield, Fla, USA.

[5] D. Hughes-Hartogs, Ensemble modem structure for imperfect transmission media, U.S. Patent 4679 227, July, 1987.

[6] S. Morosi, E. Del Re, R. Fantacci and D. Forasacchi,"Turbo-coding and bit-loading algorithms for a HomePlug-like DMT PLC system,” IEEE ISPLC 2006, pp. 227-231, March 2006.

[7] "HomePlug AV white paper," http://www.homeplug.org.

[8] K. H. Afkhamie, S. Katar, L. Yonge and R. Newman, "An overview of the upcoming HomePlug AV standard" ISPLC 2005, pp. 400-404, April 2005.

[9] E. Guerrini, G. Dell'Amico, P. Bisaglia, L. Guerrieri, "Bit-loading algorithms and SNR estimate for HomePlug AV" IEEE ISPLC '07, pp.419-424, 26-28 March 2007

[10] F.S. Muhammad, J.-Y. Baudais, J.-F. Hélard and M. Crussière, "Coded adaptive linear precoded discrete multitone over PLC channel," ISPLC'08, April 2008.

[11] N. Papandreou and T. Antonakopoulos, "Bit and Power Allocation in Constrained Multicarrier Systems: The Single-User Case," EURASIP JASP, vol 2008, 14 pages, 2008

[12] M. Tlich, A. Zeddam, F. Moulin, F. Gauthier, and G. Avril,"A broadband Powerline Channel Generator,” IEEE ISPLC07, pp. 505-510, Pisa, Italy, March 26-28, 2007.

[13] M. Crussière, J.-Y. Baudais, and J.-F. Hélard, "Adaptive linear precoded DMT as an efficient resource allocation scheme for power-line communications," Proc. of IEEE GLOBECOM'06, San Francisco, USA, November 2006.

[14] M. Crussière, J.-Y. Baudais, and J.-F. Hélard, "Adaptive SpreadSprectrum Multicarrier Multiple-Access Over Wirelines," IEEE JSAC, Vol. 24, No. 7, July 2006.

[15] B. Mashburn, H. Latchman, T. VanderMey, L. Yonge, K. Tripathi, "Signal processing challenges in the design of the HomePlug AV powerline standard to ensure co-existence with HomePlug 1.0.," IWSPAWC 2005, 5-8 June 2005.

[16] A. M. Wyglinski, F. Labeau and P. Kabal, "Bit loading with BER constraint for multicarrier systems," IEEE Trans. on Wireless Comm., vol. 4, pp. 1383-1387, July 2005. 